CARNETS DE Carnets de géographes

GÉOGRAPHES.

$1 \mid 2010$

Varia

\title{
Géopolitique et risques de marée noire en Europe
}

Julien Gardaix

\section{OpenEdition}

Journals

Édition électronique

URL : http://journals.openedition.org/cdg/2094

DOI : $10.4000 /$ cdg. 2094

ISSN : 2107-7266

Éditeur

UMR 245 - CESSMA

Référence électronique

Julien Gardaix, «Géopolitique et risques de marée noire en Europe », Carnets de géographes [En ligne], 1 | 2010, mis en ligne le 01 octobre 2010, consulté le 24 septembre 2020. URL : http:// journals.openedition.org/cdg/2094; DOI : https://doi.org/10.4000/cdg.2094

Ce document a été généré automatiquement le 24 septembre 2020.

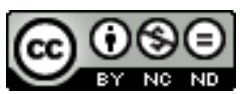

La revue Carnets de géographes est mise à disposition selon les termes de la Licence Creative Commons Attribution - Pas d'Utilisation Commerciale - Pas de Modification 4.0 International. 


\title{
Géopolitique et risques de marée noire en Europe
}

\author{
Julien Gardaix
}

Pendant plusieurs décennies, l'Europe a subi des marées noires à répétition. Ces catastrophes ont été considérées comme des « dommages collatéraux » acceptables face aux nécessités d'approvisionnement en hydrocarbures. Or le déversement spectaculaire des mazouts en mer et sur les littoraux leur donne une visibilité particulière largement reprise par les médias. Au-delà des conséquences écologiques, l'émotion est vive de voir se transformer des paysages à la symbolique forte. La situation européenne du début des années 2000 a été transformatrice : la marée noire de l'Erika puis celle du Prestige ont suscité une mobilisation sociale qui a contraint les autorités à réagir.

2 Longtemps acceptés de fait, les risques de marée noire et leur résultante étaient devenus l'emblème de paradoxes politiques, d'excès économiques et de résignation sociale. Cette donne change. Les pôles de rivalités tendent à s'équilibrer en dépassant les coopérations et les oppositions anciennes. Les interactions et les enjeux se redessinent avec les apports de la mondialisation économique sur l'ensemble des acteurs et des territoires. Une nouvelle conflictualité reconfigure les risques de marée noire en Europe. Une telle problématique est l'occasion de comprendre les marées noires de manière originale, dans la complexité de leur formation internationale, et de dégager les mécanismes lointains de la catastrophe, les solutions disponibles et les blocages dans une communauté d'États, pour aboutir à une cartographie des risques de marée noire selon des filtres géopolitiques et géoéconomiques.

3 Plusieurs étapes sont nécessaires pour mener une telle étude. Tout d'abord, le recoupement des statistiques fournies par les instituts français, européens et internationaux sur les marées noires permet d'élaborer une base de données enrichie sur les catastrophes ayant eu lieu en Europe. Son analyse met en perspective l'évolution des risques dans le temps et dans l'espace. Diverses formes de risques se succèdent avec des causes et des effets variables. Dans le même temps, les espaces littoraux et maritimes prennent une place croissante dans les sociétés, que ce soit dans l'expérience des lieux ou leur idéalisation. Un affectif puissant lie les populations à ces espaces selon 
des images normées et véhiculées par les médias. Les dernières marées noires en Europe, celles de l'Erika et du Prestige, sont dès lors inadmissibles : l'ensemble de la population, victimes directes ou non, manifeste son mécontentement contre ses pollutions, le fonctionnement du transport d'hydrocarbures et l'incurie des autorités.

Les mécanismes qui mènent aux marées noires européennes s'inscrivent dans une trame internationale opaque et complexe. Plus qu'un banal accident de navigation, les catastrophes sont issues des montages sophistiqués. Le transport maritime d'hydrocarbures est dépendant de la riche sédimentation des activités pétrolières mêlant enjeux stratégiques, géopolitiques, économiques et de plus en plus financiers. Un large travail bibliographique pose les bases des fonctionnements déséquilibrés dans ce secteur. Les coups de force, les compromis et les collusions sont nombreux pour faciliter l'approvisionnement en hydrocarbures, même en temps de crise. Aujourd'hui, la diversité des espaces en relation et la multiplication des intervenants construit un risque aux causalités mondiales et aux conséquences locales. D'internationalisées, les activités se sont mondialisées : la financiarisation des activités du transport maritime accroît les tensions sur la sécurité des navires. Les marchés du baril et du shipping sont au cœur de risques renouvelés. Finances et spéculations éloignent de la réalité des échanges physiques. Des enquêtes et des entretiens étayent ces fonctionnements et leurs effets négatifs qui mènent à la catastrophe.

Des règles contraignantes sont difficiles à imposer à l'échelle internationale. Quand elles existent, leur portée s'inscrit dans les mêmes logiques que le transport maritime. Des réglementations régionales prennent le relai face à ces dysfonctionnements. Les États-Unis servent de référence car ils ont très tôt imposé des textes de loi sévères contre les acteurs du transport maritime. La dissuasion financière en est une mesure centrale. En Europe, les divergences entre les États ont été sources de blocages pendant plusieurs décennies. Les objectifs nationaux limitent les perspectives communautaires. Certains États européens ne souhaitent pas d'entraves légales contre leurs activités d'importance telles que le transport maritime ou l'exploitation pétrolière. Le consensus est délicat à obtenir pour un risque aux causes techniques et économiques identifiables. Des compromis se dégagent et proposent dès lors des solutions partielles faisant craindre une approche superficielle des dangers et une réapparition des risques à moyen terme. L'établissement d'une gouvernance des risques de marée noire dans l'Union européenne se confronte aux enjeux propres à chaque acteur politique et économique. Cette gouvernance profite de l'impulsion donnée par la société civile (par des manifestations, la formation d'associations, des actions en justice) mécontente des marées noires à répétition. La société civile devient capable d'agir sur les autres acteurs et sur l'espace. Elle forme une " géosociété ", équivalent sociale de la géopolitique.

Cette thèse propose des clés pour faire évoluer la gestion des risques d'origines économiques, donc maitrisables par des règles, dans un cadre transnational. Une gouvernance objective des risques doit pouvoir s'établir si chaque catégorie d'acteurs est équitablement représentée. Elle reste confrontée à des enjeux de pouvoir immanents dans la mondialisation économique : les risques demeurent les révélateurs d'une concurrence mondiale et déséquilibrée entre les acteurs et les territoires.

Fiche informative 


\section{Discipline}

Géographie

\section{Directeur}

Salomon Jean-Noël

\section{Université}

Bordeaux 3

Membres du jury de thèse, soutenue en décembre 2009

- Salomon Jean-Noël, (Pr) université Bordeaux 3, Directeur de thèse

- Dauphiné André, (Pr) université de Nice Sophia-Antipolis, Examinateur

- Di Méo Guy, (Pr) uUniversité de Bordeaux, Examinateur

- Fattal Paul, (Pr) université de Nantes, Rapporteur

- Hoffmann Frédéric, (MCF) université de Bordeaux, Examinateur

- Pigeon Patrick, (Pr) université de Savoie, Rapporteur

Situation professionnelle actuelle

Rattaché à l'UMR 5185 ADES

Contact de l'auteur

juliengardaix[at]gmail.com

INDEX

Thèmes : Carnets de soutenances 